\title{
Fouille archéologique de Bunje (Novo Selo, Croatie) en 2020
}

\author{
Emmanuel Botte, Kristina Jelinčić, Audrey Bertrand, Jere Drpić, Bastien \\ Lemaire, Alexia Lattard et Nicolas Leys
}

\section{OpenEdition \\ Journals}

Édition électronique

URL : https://journals.openedition.org/baefe/3114

DOI : 10.4000/baefe.3114

ISSN : 2732-687X

Éditeur

ResEFE

Référence électronique

Emmanuel Botte, Kristina Jelinčić, Audrey Bertrand, Jere Drpić, Bastien Lemaire, Alexia Lattard et Nicolas Leys, « Fouille archéologique de Bunje (Novo Selo, Croatie) en 2020 » [notice archéologique], Bulletin archéologique des Écoles françaises à l'étranger [En ligne], Balkans, mis en ligne le 03 janvier 2022, consulté le 06 janvier 2022. URL : http://journals.openedition.org/baefe/3114 ; DOI : https:// doi.org/10.4000/baefe.3114

Ce document a été généré automatiquement le 6 janvier 2022

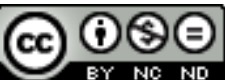

Le Bulletin archéologique des Écoles françaises à l'étranger est mise à disposition selon les termes de la Licence Creative Commons Attribution - Pas d'Utilisation Commerciale - Pas de Modification 4.0 International. 


\title{
Fouille archéologique de Bunje (Novo Selo, Croatie) en 2020
}

\author{
Emmanuel Botte, Kristina Jelinčić, Audrey Bertrand, Jere Drpić, Bastien \\ Lemaire, Alexia Lattard et Nicolas Leys
}

\section{NOTE DE L'AUTEUR}

Date précise de l'opération : 4-18 septembre 2020

Composition de l'équipe de terrain : Emmanuel Botte, Aix Marseille Univ, CNRS, CCJ, Aix-en-Provence, France, archéologue ; Jere Drpić, Institut archéologique de Zagreb, archéologue ; Bastien Lemaire, Université Paul Valéry-Montpellier 3, archéologue ;Alexia Lattard, Aix Marseille Univ, CNRS, CCJ, Aix-en-Provence, France, anthropologue ; Kristijan Majdak, Université de Zagreb, étudiant en archéologie ; Arnaud Watel, archéologue bénévole.

Partenariats institutionnels : Centre Camille Jullian, École française de Rome, Institut archéologique de Zagreb, Ministère de l'Europe et des affaires étrangères

Établissement éditeur : EFR

Établissements porteurs de l'opération : Centre Camille Jullian, École française de Rome, Institut archéologique de Zagreb

Autorité nationale présente :

Institut archéologique de Zagreb

Chroniques de l'EFR :

Bunje (Novo Selo, Croatie) 2020 (2019)

Bunje (Novo Selo, Croatie) 2019 (2018)

Bunje (Novo Selo, Croatie) 2018 (2017) 


\section{Avant-propos}

1 L'année 2020 aura marqué tous les aspects de nos travaux, et au premier plan ceux de terrain. La campagne d'avril 2020 a été annulée quelques jours avant son lancement. Quant à celle d'octobre, en raison de l'évolution sanitaire en France et en Croatie, et du fait de l'exigence par la Slovénie d'un test Covid pour les voyageurs transitant par ce pays, nous avons décidé d'anticiper la campagne au mois de septembre, et de la réaliser en effectif réduit sur une durée de deux semaines.

2 Ainsi, la plupart des objectifs fixés pour cette année se sont retrouvés décalés d'un an, et nous envisageons donc la fin de la fouille de la villa romaine de Bunje en 2021, avec une grande campagne en avril et une campagne de vérifications en octobre en vue de la publication.

\section{Introduction}

3 Le chantier de Bunje sur l'île de Brač constitue la première étape d'un programme portant sur les villae de l'Adriatique orientale, conduit par E. Botte (Centre Camille Jullian, CNRS), A. Bertrand (Université Gustave Eiffel) et K. Jelinčić (Institut archéologique de Zagreb) et intitulé « Recherches sur l'exploitation économique de la Dalmatie à l'époque romaine (II ${ }^{\mathrm{e}}$ s. av. J.-C.- II ${ }^{\mathrm{e}}$ s. apr. J.-C.) ». L'intention des auteurs de ce projet est de mesurer, à travers des opérations de fouilles et de prospections, l'impact économique de la présence romaine en Dalmatie ${ }^{1}$.

4 La première campagne menée en 2015 sur le site de Bunje (commune de Novo Selo) sur l'île de Brač était destinée à obtenir une vision d'ensemble de l'ampleur du site et de l'état de préservation de ses vestiges. Il en était ressorti un site assez étendu (les vestiges couvrant un espace de près de $15000 \mathrm{~m}^{2}$, et la villa couvrant à l'intérieur de celui-ci plus de $2500 \mathrm{~m}^{2}$ ) et dont les structures sont dans l'ensemble assez bien conservées, ce qui confirme les observations effectuées dans le cadre d'un premier rapport rédigé par K. Jelinčić en $2012^{2}$. Le site est inscrit au registre des monuments culturels sous le numéro 486 , et protégé en tant que tel auprès de la Surintendance de Split par décision du 26 septembre 1979 (sous le numéro 17/41-1979).

5 Les opérations menées sur ce site s'inscrivent dans une recherche plus large visant à clarifier les mécanismes et la chronologie de l'occupation du territoire de la Dalmatie centrale en amont et en aval de la conquête romaine de ce territoire à la fin de la période tardo-républicaine. Au sein de cette problématique, les fouilles de la villa de Bunje nous permettent de déterminer le cadre historique et environnemental qui accueille cette structure, ainsi que l'organisation de sa production.

6 En 2017 et 2018, les campagnes de fouilles nous avaient permis de faire des avancées significatives dans la compréhension du plan de la villa, en faisant apparaître la partie résidentielle (pars urbana) et la partie productive (pars rustica) de part et d'autre d'une grande cour centrale. Nous avions pu notamment compléter le plan dans les secteurs méridionaux et orientaux, faisant apparaître un bâtiment au sud-est des structures connues, ainsi qu'un pressoir à huile dans un très bon état de conservation.

7 La campagne de 2019, concentrée sur le secteur oriental de la pars rustica, n'avait pas donné d'indication claire sur la présence d'un chai, mais nous avait permis de comprendre qu'une seconde terrasse, située en contrebas de celle connue, avait fait 
l'objet d'une occupation durant l'Antiquité. La fouille du secteur 6 avait également livré la découverte d'un second pressoir, moins bien conservé que le premier, et surtout les premiers niveaux d'occupation du site, interceptés en place pour la première fois.

\section{Déroulement et objectifs de la campagne de fouille}

8 La campagne de fouilles s'est déroulée du 4 au 18 septembre 2020, sous la direction d'E. Botte (CNRS, Centre Camille Jullian) et J. Drpić (Institut archéologique de Zagreb). L'équipe de fouille était constituée de Bastien Lemaire (Université Paul ValéryMontpellier 3), Alexia Lattard (Aix-Marseille Université), Kristijan Majdak (Université de Zagreb) et Arnaud Watel.

9 La campagne a été financée par l'École française de Rome, le Ministère de l'Europe et des Affaires étrangères, le Centre Camille Jullian, le Ministère de la Culture de Croatie et la municipalité de Selca. Les mobiliers en cours d'étude et ceux considérés comme importants pour la datation et l'histoire du site ont été consignés à l'Institut archéologique de Zagreb. L'étude archéozoologique est réalisée par Siniša Radović (Académie des sciences et des arts à Zagreb) ; l'étude anthracologique par Christophe Vaschalde (Université Paul Valéry-Montpellier 3); l'étude micro-morphologique des sols par Cristiano Nicosia (Université de Padoue); l'étude du verre par Bartul Siljeg (Institut archéologique de Zagreb); les études anthropologiques par Mario Novak (Institut d'anthropologie de Zagreb) et Alexia Lattard (Aix-Marseille Université) ; les études numismatiques par Anja Bertol (Université de Zagreb) ; les analyses chimiques par Nicolas Garnier (Laboratoire Nicolas Garnier). Nicolas Leys (Sorbonne Université), responsable de la photogrammétrie sur le site, a fait une modélisation 3D du site et des principaux blocs de pierre. Les relevés topographiques et les plans ont été réalisés par Kristina Jelinčić et Jere Drpić. L'étude du mobilier céramique a été réalisée par Emmanuel Botte.

10 Par ailleurs, nous avons reçu en 2020 la visite de M. l'Ambassadeur de France, M.G. Veyssière, que nous remercions pour son soutien à notre mission.

11 Les objectifs de la campagne étaient concentrés sur les secteurs 4 et 11. Le premier correspond à la terrasse inférieure, dont il nous faut encore déterminer la nature de l'occupation et établir sa relation chronologique avec la terrasse principale. D'autre part, un sondage a été ouvert dans le secteur 11 situé à l'est de la villa afin d'interpréter la structure bâtie découverte en 2018 (fig. 1 et 2). 
Fig. 1. Vue par drone de la villa au début de la campagne.

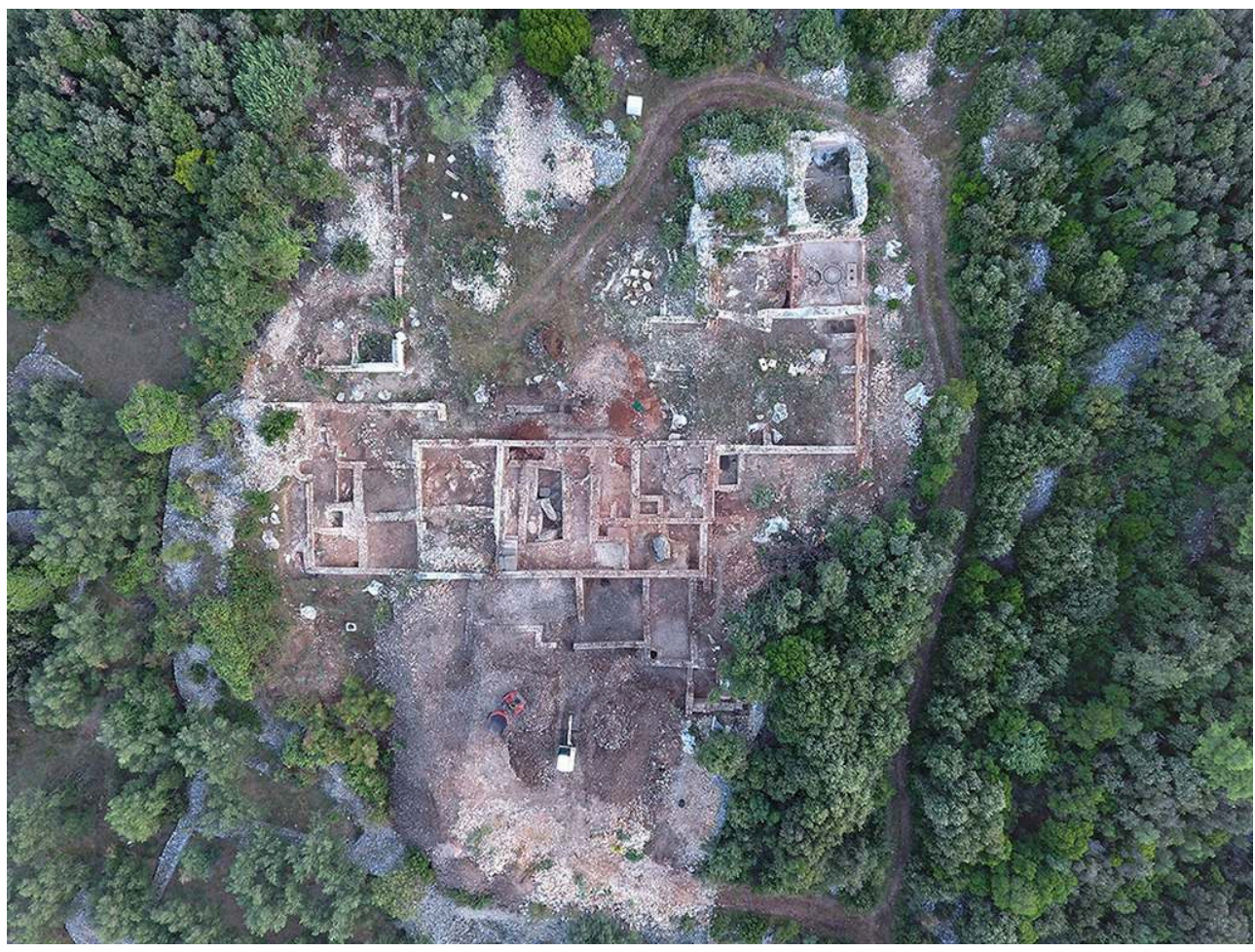

(C)E. Botte.

Fig. 2. Plan de la villa avec les secteurs fouillés durant cette campagne.

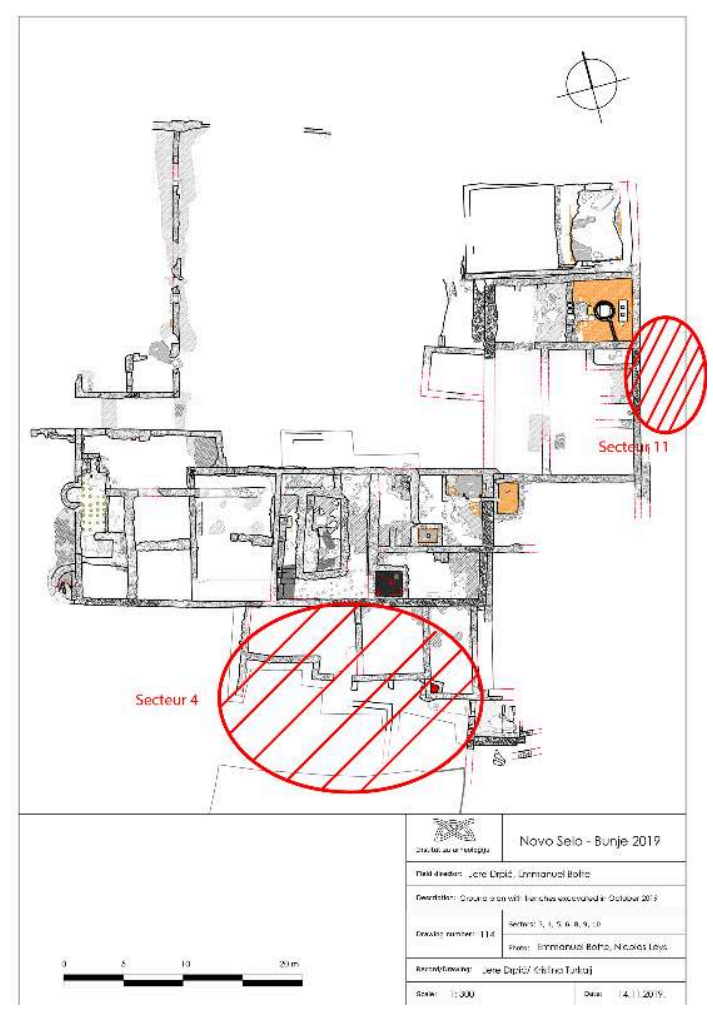

Plan J. Drpić. 


\section{Résultats}

\section{Secteur 4}

Lors d'une campagne précédente, nous avions mis au jour le départ d'un mur (MR 7023) appuyé au mur méridional du secteur 6 (MR 6009). Nous en avions alors déduit qu'il existait une terrasse inférieure, dont l'organisation et la chronologie restaient à définir. Une partie de la campagne a donc été dédiée au dégagement de cet espace (fig. 3). Les résultats en sont pour le moment mitigés car nous avons surtout passé l'essentiel du temps à vider les pièces qui étaient remplies de pierres sur parfois plus de deux mètres de hauteur. Il en ressort, dans l'état actuel de conservation, plusieurs pièces presque totalement vides de mobilier, ce qui pour le moment rend la datation de ces espaces délicate. Néanmoins, le fait qu'aucune structure de production ne soit présente nous amène à envisager l'hypothèse que cette terrasse ait eu uniquement, ou en tout cas principalement, une vocation résidentielle et éventuellement de stockage. La partie méridionale de cette terrasse a complètement disparu suite à des travaux de terrassement, et pour l'heure ses dimensions sont d'au moins $20 \mathrm{~m}$ de long sur $14,5 \mathrm{~m}$ de large, avec à ce jour 7 pièces visibles, mais toutes n'ont pas été dégagées.

Fig. 3. Vue d'ensemble de la villa, avec dans la partie basse la terrasse inférieure en cours de fouille.

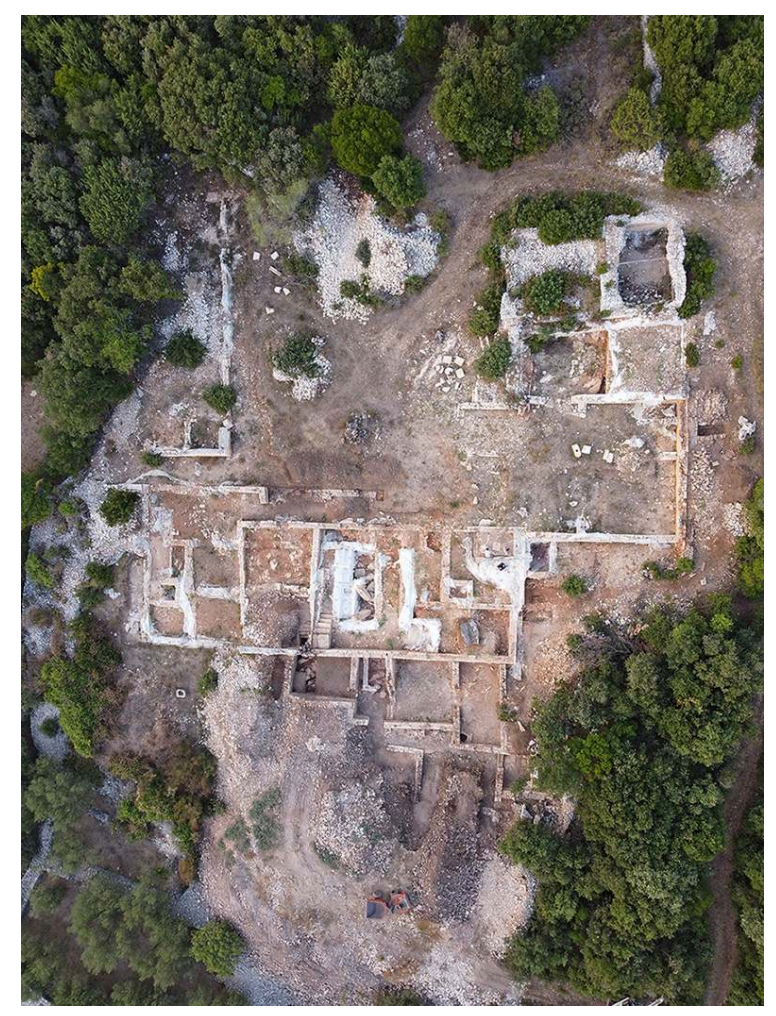

(c) B. Botte.

13 Nous avons pu observer que de manière générale tout le mobilier découvert dans les espaces de cette terrasse inférieure ne semble pas antérieur au $\mathrm{IV}^{\mathrm{e}}$ siècle de notre ère, ce qui nous incite à penser que celle-ci n'aurait été aménagée que dans la dernière grande phase d'occupation de la villa. Dans ce cas, seul le bassin BS 4005 était présent 
antérieurement. Sa fonction, dans un espace où aucune autre structure n'est contemporaine, et avec une orientation qui ne fonctionne avec aucune autre structure bâtie de la villa, reste à déterminer.

\section{Secteur 11}

Le secteur 11 se trouve à l'est de la villa, accolé contre son mur de limite orientale. Nous y avons consacré une partie de la campagne car lors des dégagements de ce secteur en 2018 une grande structure bâtie était apparue (SB 11003) (fig. 4). Dans celle-ci apparaissaient des fragments de béton de tuileau et il nous fallait donc comprendre la destination de cette construction. La villa étant construite à flanc de colline, nous nous demandions s'il pouvait s'agir de la couverture d'un égout destiné à détourner les eaux pluviales pour qu'elles ne rentrent pas dans la villa. Suite au nettoyage de cette année, il semblait que nous avions affaire à la couverture en berceau d'une structure qui restait à déterminer (canalisation ou sépulture). Nous avons procédé à un nettoyage de la structure, dont les dimensions sont de $2 \mathrm{~m}$ de large sur au moins 3,20 $\mathrm{m}$ de long. Un sondage réalisé dans l'arrachement d'un arbre en son centre ne nous a pas permis de comprendre la nature de cette construction. En effet, aucun mur n'apparaît en dessous et le niveau 11003 s'apparente plus à un hérisson coulé directement sur la terre, et dont l'ensemble de l'élévation a été détruit.

Fig. 4. Vue depuis le sud-ouest de la structure bâtie SB 11003, et de la sépulture SP 11009 installée sur son flanc sud.

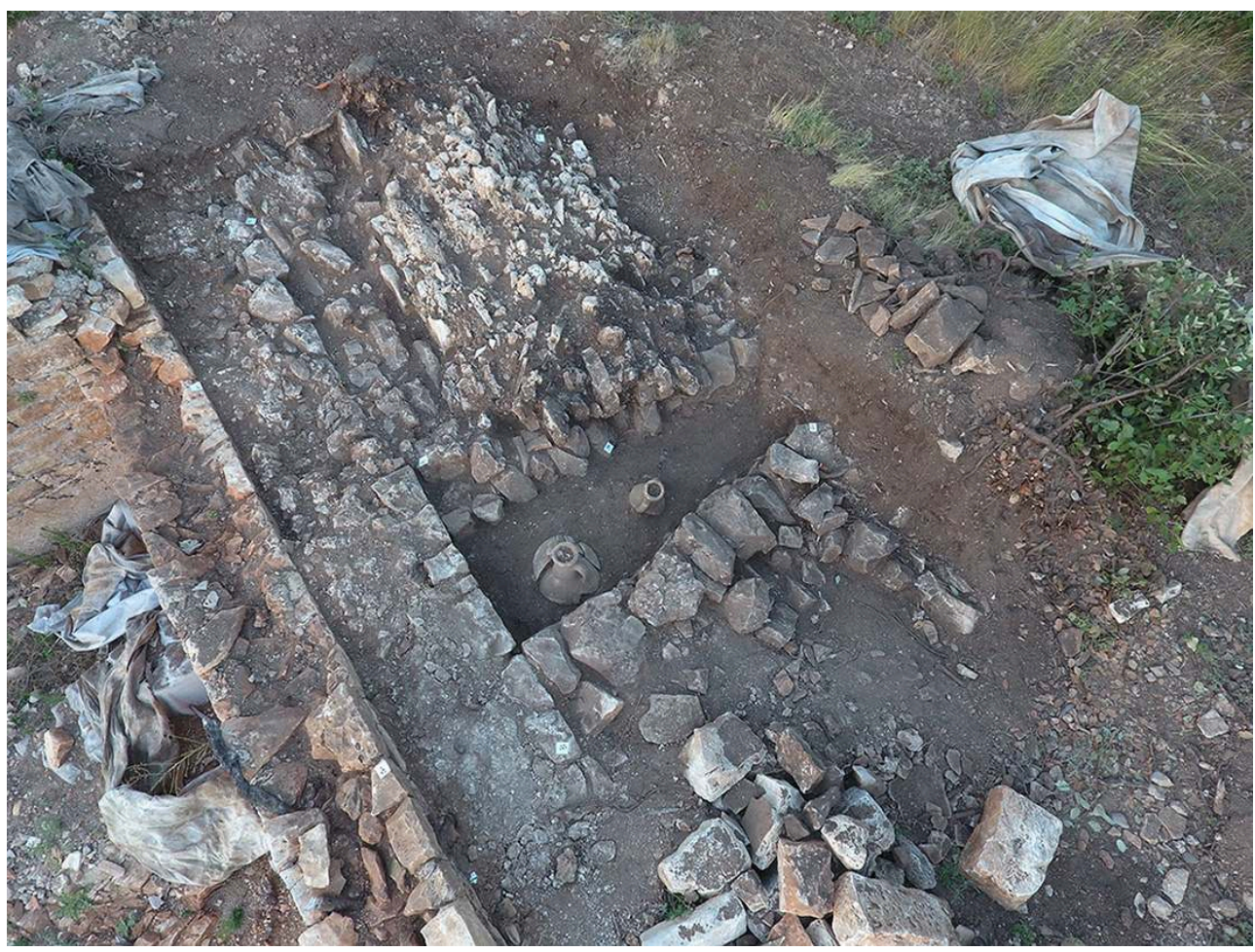

(c)E. Botte.

Néanmoins, la découverte d'une sépulture de l'Antiquité tardive sur son flanc sud et la suspicion d'autres sépultures autour de cette structure nous invite à lui attribuer une vocation funéraire. 
Nous avons en effet mis au jour une sépulture (SP 11009) dont la datation ne pose pas de difficultés. Elle est installée au sud de la structure bâtie 11003, dans une fosse aménagée sur le substrat. Au fond de celle-ci ont été installées deux amphores africaines de type Keay 61 (11008 et 11013) (fig. 5), dont la production est attestée entre la fin du VI et la première moitié du VII ${ }^{e}$ siècle de notre ère ${ }^{3}$. Les cols de ces amphores (11002 et 11005) ont été soigneusement sciés afin de pouvoir y disposer le corps d'un enfant âgé entre 9 et 11 ans (fig. 6). Les cols ont par la suite été installés sur le sommet de la fosse, peut-être comme conduits à libation (fig. 7). La fouille et l'étude anthropologique ont été menées par l'anthropologue A. Lattard (Aix Marseille Université). Durant la fouille, il est apparu que le défunt détenait comme unique mobilier un bracelet en métal qui a révélé des restes de textile (fig. 8). Celui-ci appartenait soit à un linceul dans lequel l'enfant était enveloppé, soit aux vêtements portés par le défunt. L'objet a été prélevé et sera soumis pour étude à un spécialiste du textile.

Fig. 5. Vue depuis le sud de la sépulture SP 11009, aménagée dans les amphores 11008 et 11013.

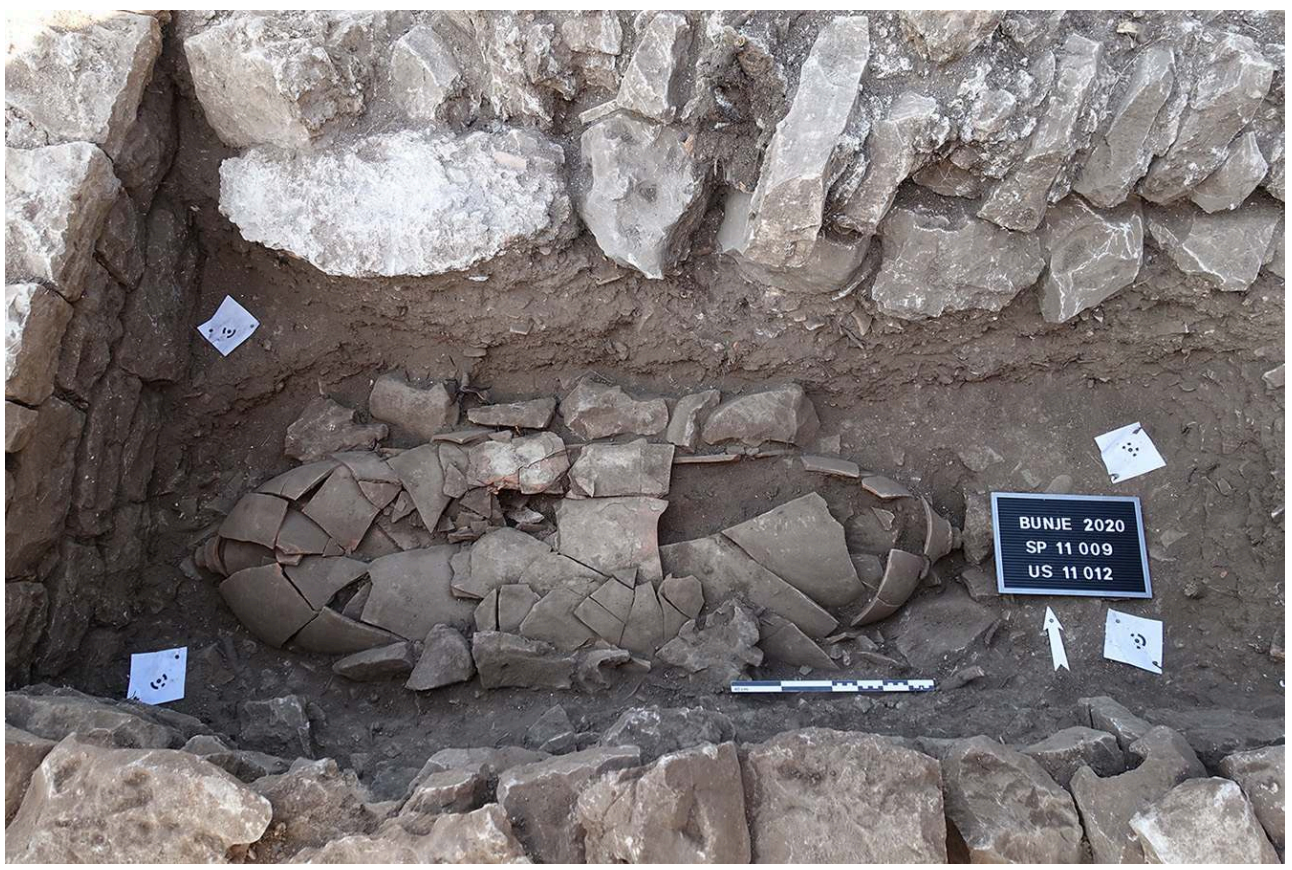

(C)E. Botte. 
Fig. 6. Vue depuis le sud des restes osseux de la sépulture SP 11009.

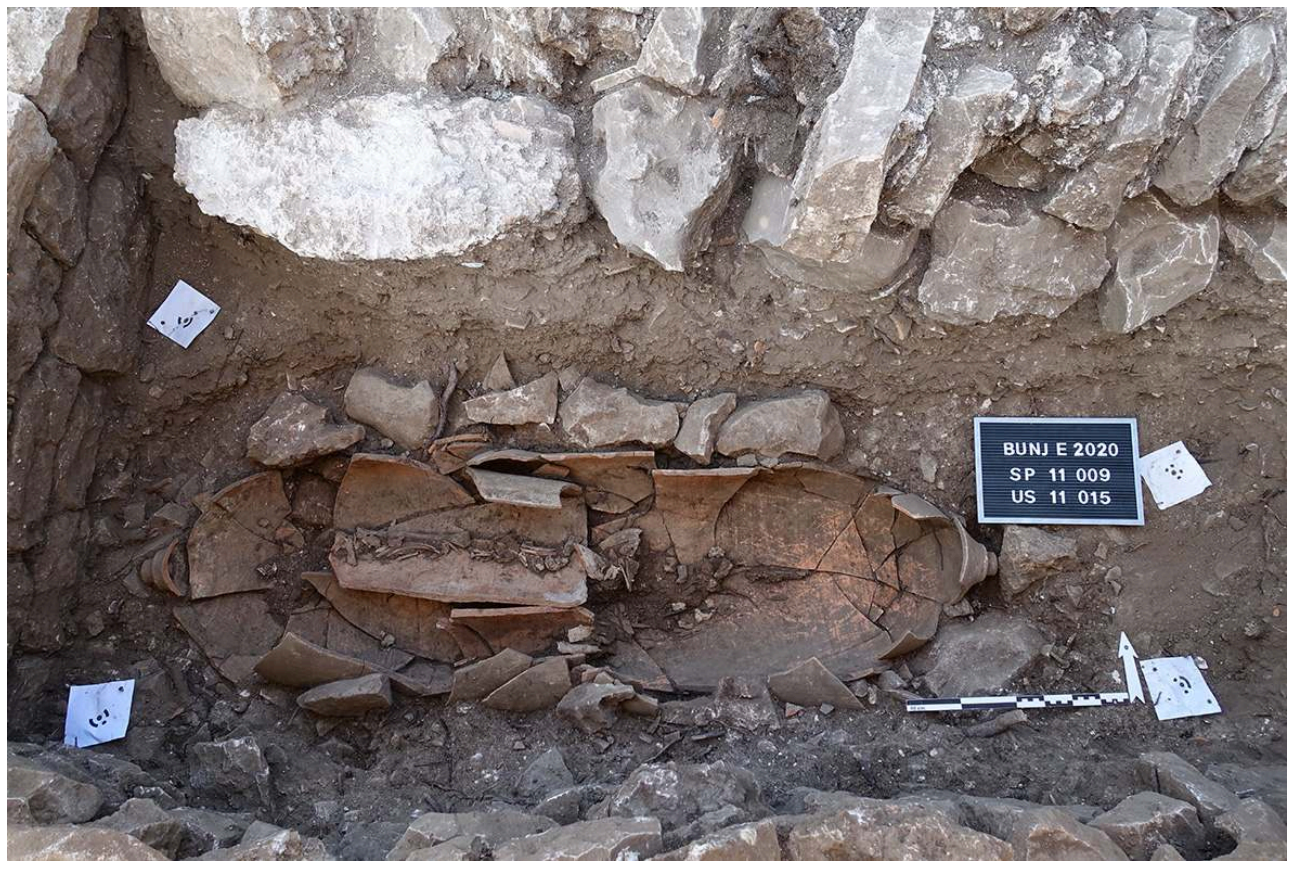

(C)E. Botte.

Fig. 7. Vue depuis l'est des cols d'amphores 11002 et 11005 .

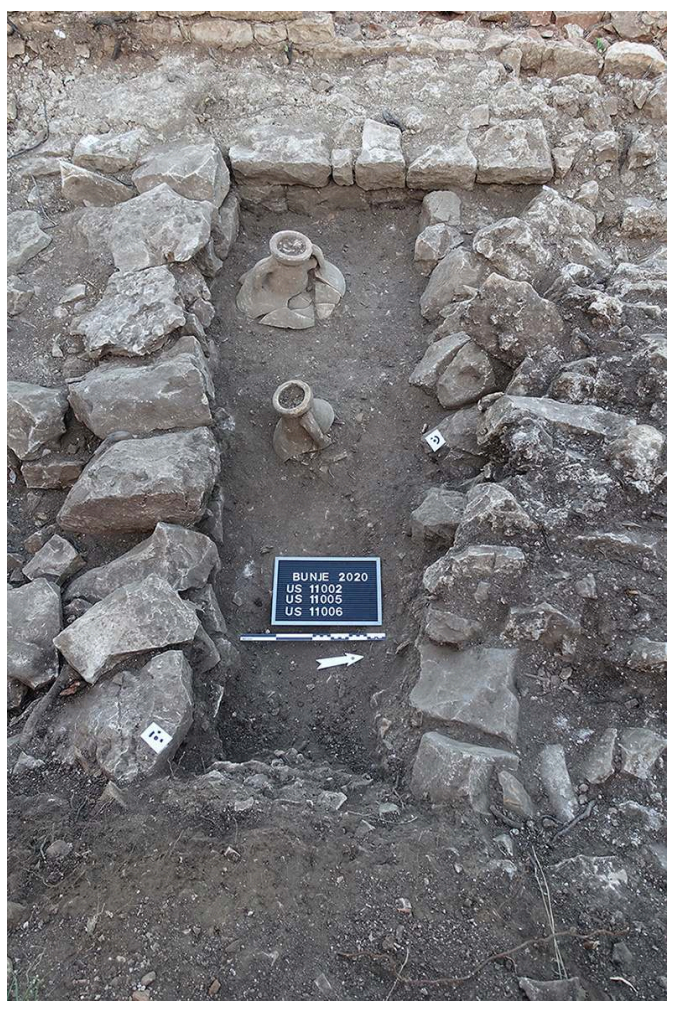

(c)E. Botte. 
Fig. 8. Restes de tissu contre le bracelet découvert dans la sépulture SP 11009.

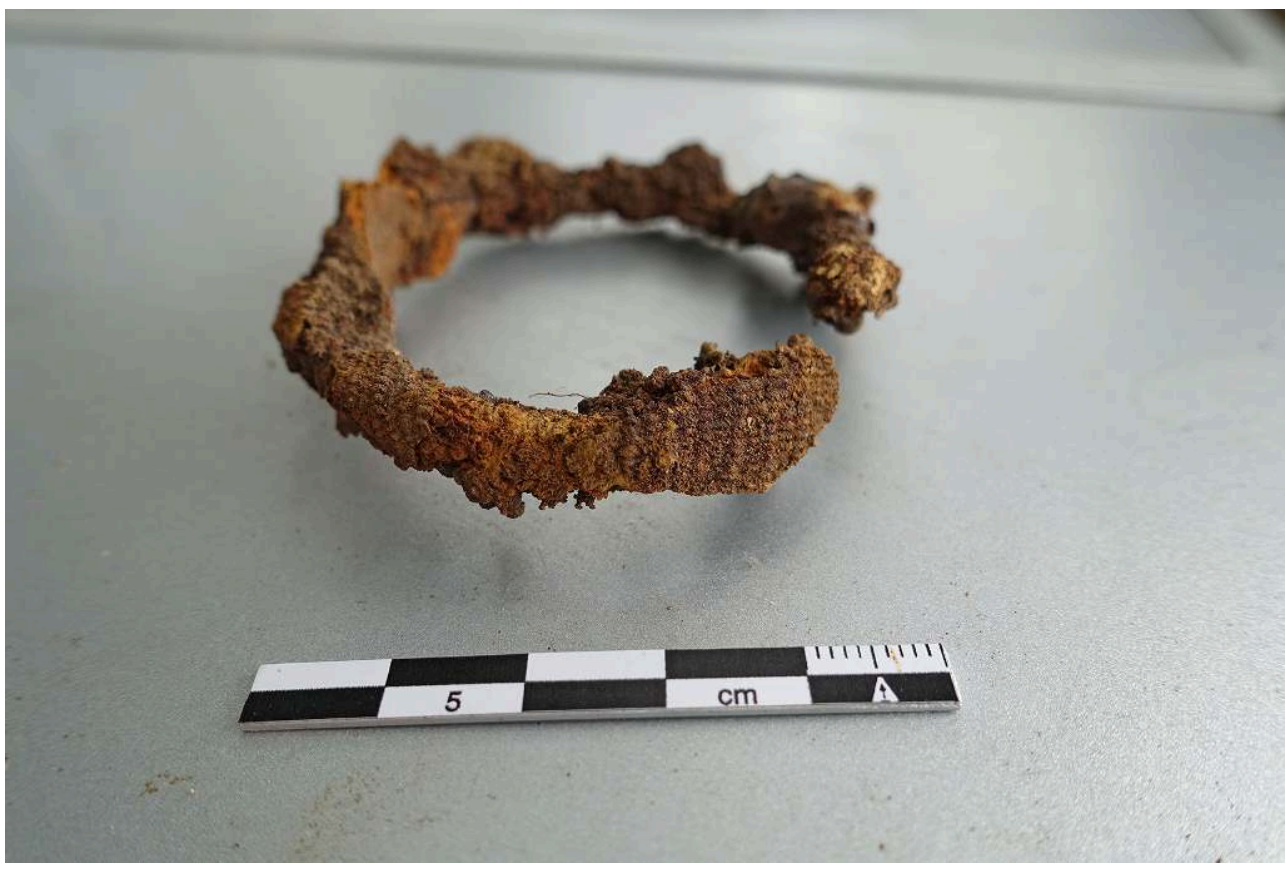

(C)E. Botte.

\section{Bilan de la campagne et programmation 2021}

17 Au terme de cette campagne, organisée en urgence mais qui a malgré tout livré des résultats intéressants, nous estimons avoir rempli la plupart des objectifs que nous nous étions fixés au début de ce programme en 2016 (fig. 9 et 10). La fouille de la villa de Bunje sur l'île de Brač constituait la première étape d'un projet à grande échelle géographique, dans lequel celle-ci devait nous permettre d'évaluer la faisabilité de notre projet. L'idée était en effet d'observer les modalités d'occupation du territoire au lendemain de la conquête de cet espace par les Romains et de la fondation des premières colonies dans la seconde moitié du Ier siècle avant notre ère. 
Fig. 9. Vue par drone de la villa à la fin de la campagne.

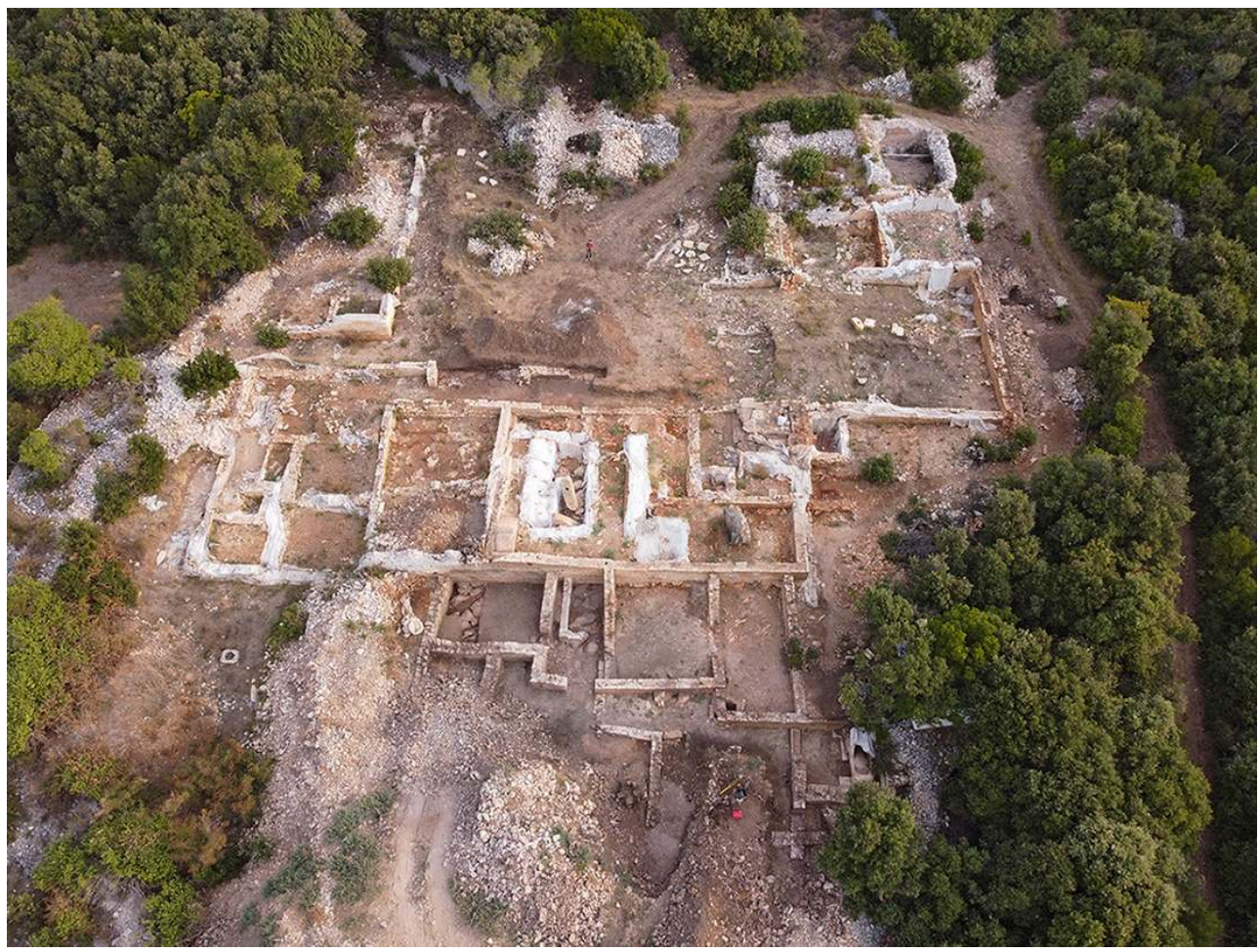

(C)E. Botte.

Fig. 10. Plan de la villa à la fin de la campagne.

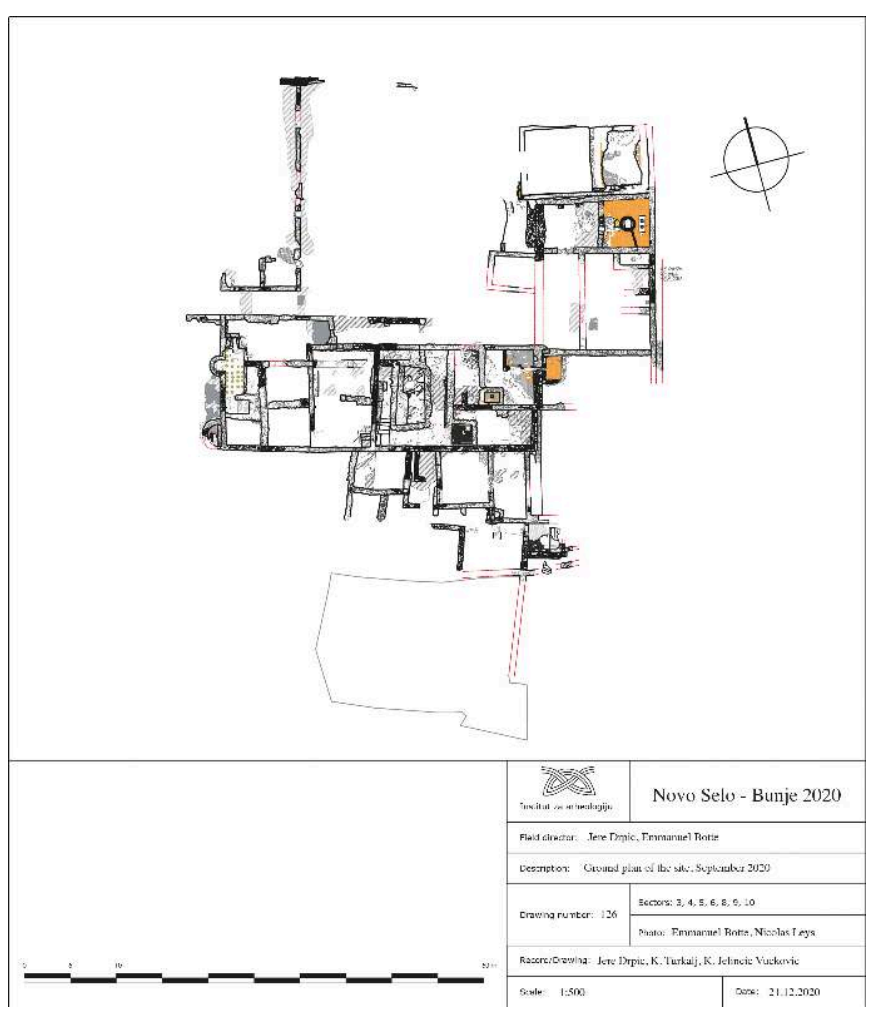

Plan J. Drpić. 
18 les dernières vérifications auraient pu être réalisées durant la campagne d'automne. Il nous reste donc une dernière grande campagne à réaliser, si possible en avril 2021, de sorte que la campagne de l'automne 2021, si elle s'avère nécessaire, ne concerne que des vérifications, sans fouille, en vue de la publication. Se tiendront en parallèle les études du mobilier céramique, qui a livré depuis le début des fouilles environ 10.000 fragments, ainsi que du verre, de la faune et des enduits peints.

La suite du programme nous porte de manière naturelle sur le territoire de l'île de Hvar. Si l'année 2021 sera consacrée à la finalisation du programme de fouilles de la villa romaine de Bunje, nous souhaitons en parallèle mettre en place un programme de prospections géophysiques et magnétiques sur quatre sites, un situé à Brač et les trois autres sur l'île de Hvar. Ces opérations seront réalisées par une équipe de géophysiciens du CEREGE (CNRS-AMU, Aix-en-Pce), composée de Y. Quesnel, P.-E. Mathé et M. Uehara, qui viendront sur le terrain en avril-mai 2021. C'est en fonction des résultats de ces prospections que nous déciderons sur quel site nous souhaitons nous investir. L'île de Hvar est particulièrement intéressante car elle nous permettra de répondre à des questions de survivances et changements entre les occupations grecque et romaine de l'île, ce que nous ne pouvons réaliser à Brač puisqu'elle n'offre pas, en l'état de la documentation, de sites d'occupation par des colons grecs, que ce soit à l'époque classique ou hellénistique.

\section{BIBLIOGRAPHIE}

BONIFAY 2004

Michel Bonifay, Études sur la céramique romaine tardive d'Afrique, Oxford, Archaeopress, BAR International Series 1301, 2004.

BOTTE et al. 2016

Emmanuel Botte, Audrey Bertrand, Kristina Jelinčić, « Bunje (Novo Selo, Croatie) », Chronique des activités archéologiques de l'École française de Rome [En ligne], Balkans, mis en ligne le 10 mars 2016. DOI : $10.4000 /$ cefr.1519.

BOTTE, JELINČIĆ 2017

Emmanuel Botte, Kristina Jelinčić, « Bunje (Novo Selo, Croatie) », Chronique des activités archéologiques de l'École française de Rome [En ligne], Balkans, mis en ligne le 16 mai 2017. DOI : $10.4000 /$ cefr.1723.

BOTTE et al. 2019

Emmanuel Botte, Audrey Bertrand, Kristina Jelinčić, Nicolas Leys et Antoine Boisson, « Bunje (Novo Selo, Croatie), campagnes 2017-2018 ", Chronique des activités archéologiques de l'École française de Rome [En ligne], Balkans, mis en ligne le 23 janvier 2019, consulté le 20 avril 2019. DOI : 10.4000/cefr.2419.

Bulletin archéologique des Écoles françaises à l'étranger , Balkans 
JELINČIĆ 2012

Kristina Jelinčić, Izvješće arheološkom rekognosciranju i snimanju lokaliteta Novo Selo Bunje (18.-19. travnja 2012.), Institut za arheologiju, Zagreb, 2012.

\section{NOTES}

1. Pour un résumé des résultats des campagnes précédentes, voir BOTTE et al. 2016 ; BOTTE, JELINČIĆ 2017 ; BOTTE et al. 2019.

2. JELINČIĆ 2012.

3. BONIFAY 2004, p. 140-141.

\section{INDEX}

lieux https://ark.frantiq.fr/ark:/26678/pcrtuF5kY9G9s0 sujets https://ark.frantiq.fr/ark:/26678/pcrtLEIWWX4Z3O, https://ark.frantiq.fr/ark:/26678/ pcrt3mTg9h3RFE, https://ark.frantiq.fr/ark:/26678/pcrttvE2YZwZu6, https://ark.frantiq.fr/ ark:/26678/pcrtwouxfBaTr5, https://ark.frantiq.fr/ark:/26678/pcrt795b632nWw

Thèmes : EFR

Année de l'opération : 2020

chronologie https://ark.frantiq.fr/ark:/26678/pcrtxT02uJOogm

\section{AUTEURS}

\section{EMMANUEL BOTTE}

Aix Marseille Univ, CNRS, CCJ, Aix-en-Provence, France

\section{KRISTINA JELINČIĆ}

Institut archéologique de Zagreb

\section{AUDREY BERTRAND}

Université Gustave Eiffel

\section{JERE DRPIĆ}

Institut archéologique de Zagreb

\section{BASTIEN LEMAIRE}

Université Paul-Valéry Montpellier 3

\section{ALEXIA LATTARD}

Aix Marseille Univ, CNRS, CCJ, Aix-en-Provence, France 
NICOLAS LEYS

Sorbonne Université 